\title{
Calculation of the Coupling Coefficient of Twin-Core Fiber Based on the Supermode Theory with Finite Element Method
}

\section{Tianhao Zhao*, Wenhua Ren, Tingya Yin, Fan Wang}

Key Laboratory of All Optical Network \& Advanced Telecommunication Network of EMC, Institute of Lightwave Technology, Beijing Jiaotong University, Beijing, China

Email: *zhaotianhao@bjtu.edu.cn

How to cite this paper: Zhao, T.H., Ren, W.H., Yin, T.Y. and Wang, F. (2021) Calculation of the Coupling Coefficient of Twin-Core Fiber Based on the Supermode Theory with Finite Element Method. Optics and Photonics Journal, 11, 402-411. https://doi.org/10.4236/opj.2021.118029

Received: January 29, 2021 Accepted: August 27, 2021 Published: August 30, 2021

\begin{abstract}
Currently, coupled mode theory (CMT) is widely used for calculating the coupling coefficient of twin-core fibers (TCFs) that are used in a broad range of important applications. This approach is highly accurate for scenarios with weak coupling between the cores but shows significant errors in the strong coupling scenarios, necessitating the use of a more accurate method for coupling coefficient calculations. Therefore, in this work, we calculate the coupling coefficients of TCFs using the supermode theory with finite element method (FEM) that has higher accuracy than CMT, particularly for the strong coupling TCF. To investigate the origin of the differences between the results obtained by these two methods, the modal field distributions of the supermodes of TCF are simulated and analyzed in detail.
\end{abstract}

\section{Keywords}

Coupling Coefficient, Twin-Core Fiber, Supermode Theory, Finite Element Method, Coupled Mode Theory

\section{Introduction}

Recent years have seen a dramatic increase in the attention devoted to research on the twin-core fiber (TCF) with two parallel cores around the center of the fiber [1] [2] [3] [4]. The coupling effect between the two cores caused by the evanescent light field can realize multi-selection function for light power, wavelength, polarization, and other properties [5] [6] [7] [8] [9]. Compared to the conventional fiber, TCF has a broader range of possible applications in filters and directional couplers [10] [11] [12] [13]. For these applications, the coupling 
coefficient is one of the most important parameters describing the optical coupling between the cores [14] [15] [16] [17]. Therefore, an accurate calculation of the coupling coefficient is highly important for the design and applications of TCFs. The conventional methods for calculating the coupling coefficient of the TCF are based on the coupled mode theory (CMT) and may only be applicable for weak coupling scenarios and give erroneous results in the case of strong coupling between the cores [18].

In this paper, we focus on the study of the coupling coefficient of the TCF with two symmetric single-mode cores. The rest of the paper is organized as follows. In Section 2, the coupling coefficients of the TCF are investigated in detail for different gap sizes (edge-to-edge distance between the adjacent cores), core radii, and relative index differences using the supermode theory with finite element method (FEM). Moreover, to prove the importance and necessity of using the method based on the supermode theory with FEM for the calculation of the coupling coefficient, it is verified that the analytical expression based on CMT leads to obvious errors in the calculations for strong coupling scenarios through the comparison of the coupling coefficients obtained by CMT with the results obtained by the supermode theory with FEM calculations. In Section 3, the modal field distribution of the TCF is also analyzed using CMT and the supermode theory with FEM calculations to investigate the origin of the errors of the CMT analytical expression for the coupling coefficient in the strong coupling scenario. It is concluded that the method for the calculation of the coupling coefficient between modes in a TCF based on the supermode theory with FEM described in this paper is effective and accurate in both strong and weak coupling scenarios, and can be extended to the directional couplers and filters.

\section{Theory}

The general form of TCF with two identical cores supporting only the fundamental $\mathrm{HE}_{11}$ mode is illustrated in Figure 1. The two cores are arranged as shown in Figure 1 where $a$ is the core radius, $n_{1}$ and $n_{0}$ are the refractive indices of the core and cladding of the TCF, respectively, and $d_{g}$ is the size of the gap between the adjacent cores. In addition, the operational wavelength is $\lambda$ and the relative index difference is $\Delta$ [19].

According to the supermode theory, first-order and second-order supermodes are present in a twin-core single-mode fiber, and their propagation constants are
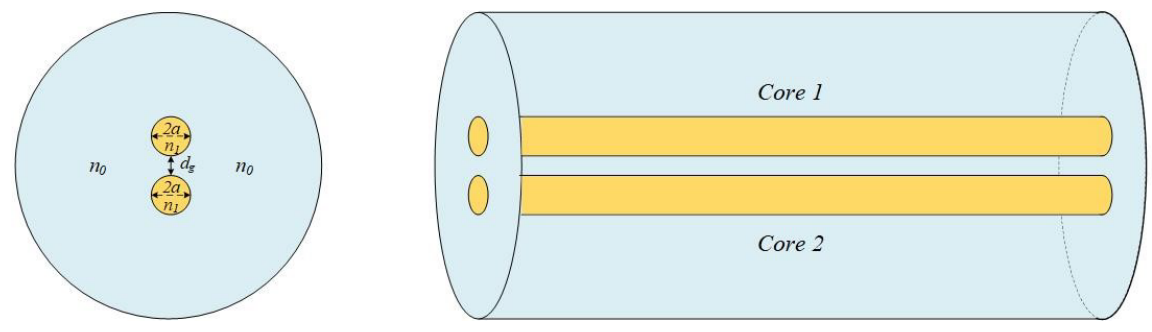

Figure 1. Schematic of the TCF structure with two identical cores. 
$\beta_{1}$ and $\beta_{2}$, respectively. The coupling length is defined as the shortest fiber length that is necessary for the incident light power to be transferred completely from one core to another core. For supermode theory, the coupling length $L$ can be calculated as [20] [21]

$$
L=\frac{\pi}{\beta_{1}-\beta_{2}}=\frac{\pi}{2 \kappa_{e}} .
$$

Based on Equation (1), the effective coupling coefficient $\kappa_{e}$ can be expressed as

$$
\kappa_{e}=\frac{\beta_{1}-\beta_{2}}{2} .
$$

For a twin-core optical fiber with two symmetrical cores, the relation between the coupling coefficient $\kappa$ and the effective coupling coefficient $\kappa_{e}$ is

$$
\kappa=\kappa_{e} .
$$

We use FEM calculations as implemented by the commercial COMSOL Multiphysics software to model and simulate the TCF and obtain accurate values of $\beta_{1}$ and $\beta_{2}$, and then use the Equations (2) and (3) to obtain an accurate value of $\kappa$.

Moreover, to verify the importance of the method based on the supermode theory with FEM for calculating the coupling coefficients in strong coupling conditions, we compare the coupling coefficients between the two fundamental modes in the TCF with different gap sizes $d_{g}$, core radii $a$ and relative index differences $\Delta$ obtained using Equations (2) and (3) based on the supermode theory with FEM with those obtained CMT.

According to CMT, the analytical expression for the calculation of the coupling coefficient is [18] [22]

$$
\kappa=\frac{\omega \varepsilon_{0} \int_{-\infty}^{\infty} \int_{-\infty}^{\infty}\left(n_{1}^{2}-n_{0}^{2}\right) E_{1}^{*} \cdot E_{2} d x d y}{\int_{-\infty}^{\infty} \int_{-\infty}^{\infty} u_{z} \cdot\left(E_{1}^{*} \times H_{1}+E_{1} \times H_{1}^{*}\right) d x d y},
$$

In order to make cores in TCF support only the fundamental $\mathrm{HE}_{11}$ mode, the basic structural parameters are fixed as the following: $a=3.5 \mu \mathrm{m}, d_{g}=2 \mu \mathrm{m}, n_{0}=$ 1.444, $\lambda=1550 \mathrm{~nm}$, and $\Delta=0.25 \%$, unless some parameter is investigated and varied. The coupling coefficients for different $d_{g}$, and $\Delta$ together with the relative differences between the CMT and the supermode theory with FEM values are shown in Figures 2-4, respectively.

The coupling coefficients between the modes in the TCF and the corresponding relative error are shown as a function of $d g$ in Figure 2(a) and Figure 2(b), respectively. Figure 2(a) shows that the coupling coefficient increases with the decreasing $d_{g}$ owing to the fact that the decrease in $d_{g}$ leads to the increase of the effective modal field intensity in Equation (4), and it is observed a discrepancy between the coupling coefficient obtained by CMT and that obtained by the supermode theory with FEM for small $d_{g}$. Similarly, the relative error $R_{e}$ increases with the decreasing gap size in Figure 2(b). Thus, as $d_{g}$ decreases, the strength of the mode coupling between the cores increases, along with the increasing 


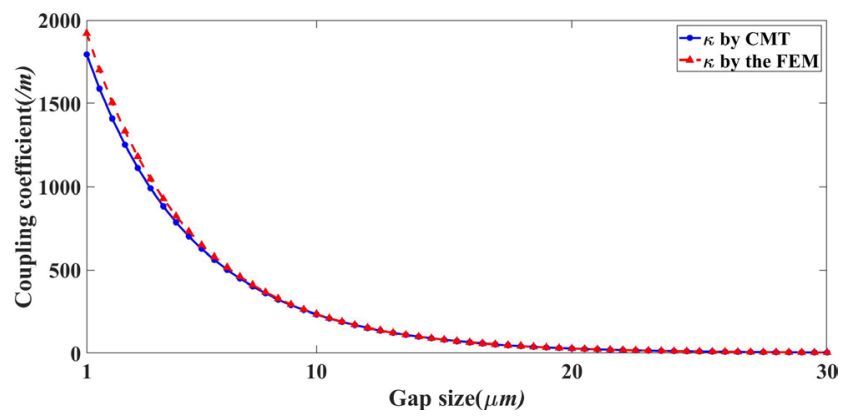

(a)

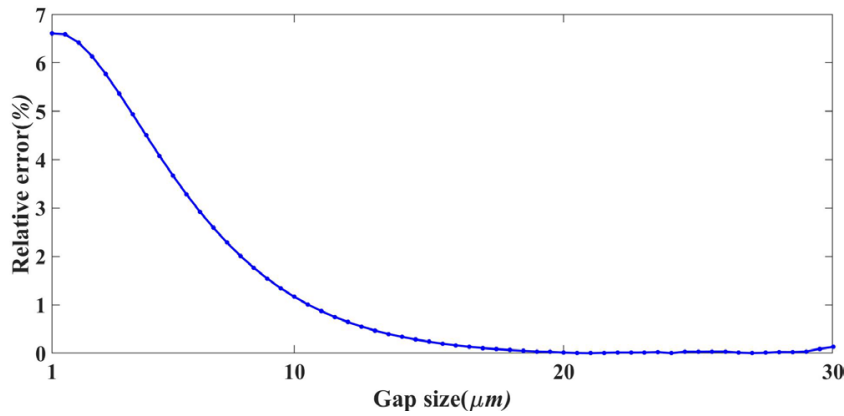

(b)

Figure 2. (a) Coupling coefficient of two fundamental $\mathrm{HE}_{11}$ modes as a function of the gap size, for a working wavelength of $1.55 \mu \mathrm{m}$, core radius of $3.5 \mu \mathrm{m}$, cladding index of 1.444 , and relative index difference of $0.25 \%$. (b) Corresponding relative errors as a function of the gap size.

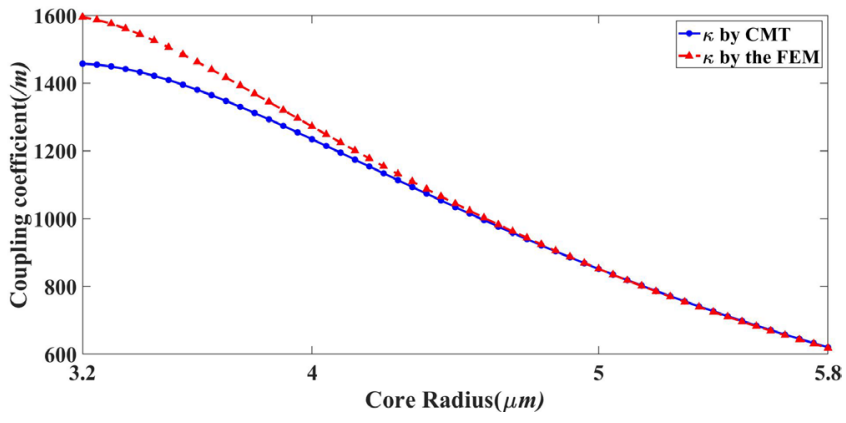

(a)

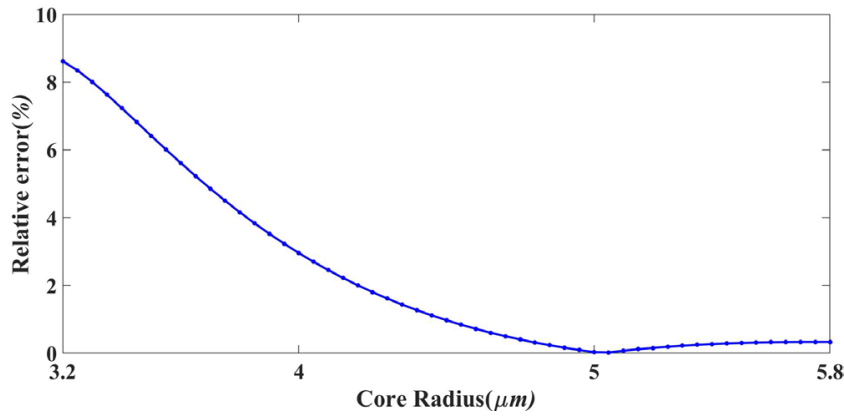

(b)

Figure 3. (a) Coupling coefficient of two fundamental $\mathrm{HE}_{11}$ modes as a function of the core radius, with gap size of $2 \mu \mathrm{m}$, other parameters are the same as those used to obtain Figure 2. (b) Corresponding relative errors as a function of the core radius. 


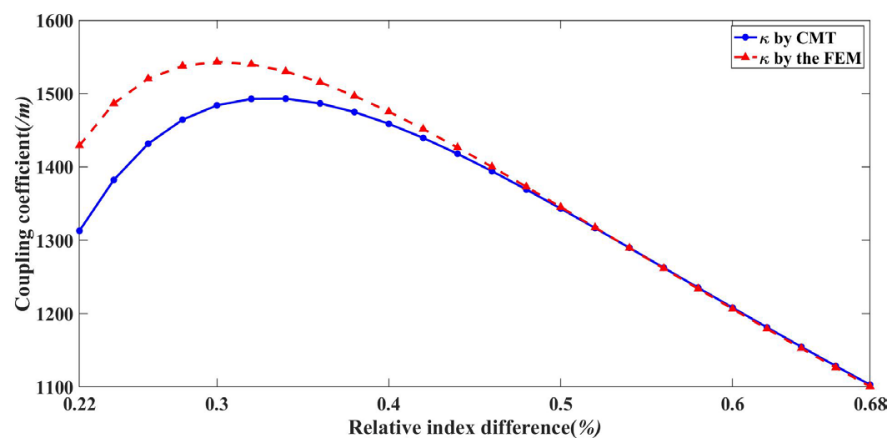

(a)

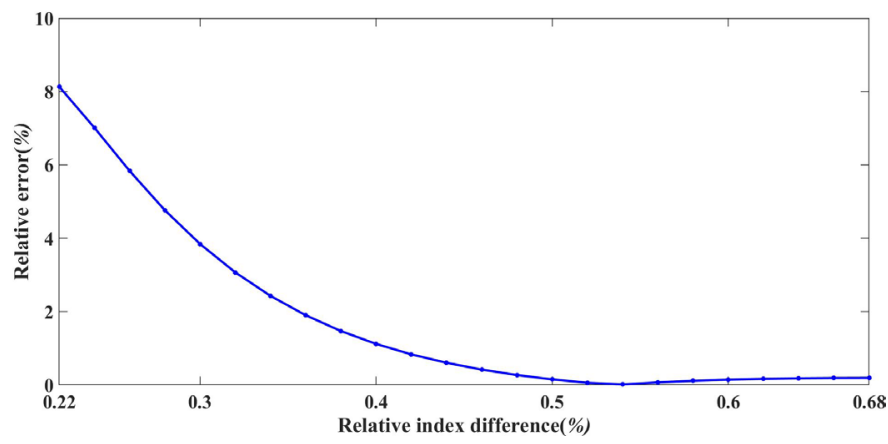

(b)

Figure 4. (a) Coupling coefficient of two fundamental $\mathrm{HE}_{11}$ modes as a function of the relative index difference, other parameters are the same as those used to obtain Figure 2 and Figure 3. (b) Corresponding relative errors as a function of the relative index difference.

relative error. For example, it is observed that the $R_{e}$ increases to approximately $6.6 \%$ when $d_{g}$ decreases to 0 .

Figure 3 shows coupling coefficients and $R_{e}$ as a function of the core radius a. It can be seen from Figure 3(a) that the coupling coefficient increases with the decreasing a due to the increasing effective modal field intensity, while there is an obvious discrepancy between the coupling coefficients obtained by the two methods particularly for small a. And Figure 3(b) shows that $R_{e}$ overall increases with the decreasing $a$. For a fixed gap size, less optical power is confined in the core region for a smaller core radius, strengthening the mode coupling between the cores. $R_{e}$ increases with decreasing $a, R_{e}$ is greater than $8 \%$ when the $a$ is approximately $3.2 \mu \mathrm{m}$. In addition, it is shown from Figure $3(\mathrm{~b})$ that $R_{e}$ slightly increases with an increasing core radius of bigger than $5 \mu \mathrm{m}$.

The results of the analysis of the impact of the relative index difference $\Delta$ on the coupling coefficients between the modes in the TCF are given in Figure 4. It is observed that the coupling coefficient firstly increases and then decreases with the decreasing $\Delta$, because in Equation (4), the effective modal field intensity increases dominantly and then the coupling coefficient decreases with the decreasing $\Delta$ since that at this time the $\left(n_{1}^{2}-n_{0}^{2}\right)$ decreases significantly as the $\Delta$ decreases. It is also shown from Figure 4 (b) that $R_{e}$ slightly increases with an increasing relative index difference of bigger than $0.54 \%$. 
In summary, the simulation results show that there is a significant discrepancy between the coupling coefficient value obtained by CMT and that obtained by the supermode theory with FEM for the case of strong coupling between cores, e.g., when either the gap size $d_{g}$, core radius a, or relative index difference $\Delta$ are quite small, demonstrating that the results obtained using CMT will be erroneous in strong coupling scenarios. In this case, the supermode theory with FEM approach should be used for a more accurate calculation of the coupling coefficient.

\section{Simulation and Discussion}

It can be seen from Equation (4) that according to CMT, $\kappa$ depends on the modal field distribution. In addition, the modal field distribution is an important characteristic for the supermodes that determines the effective area of the supermodes related to the nonlinear properties of TCF. To study the origin of the observed errors for the results obtained using the CMT analytical expression in the strong coupling scenario described above, we simulated the modal field distributions of the first-order supermode and the second-order supermode in the TCF with two identical single-mode cores using CMT and the supermode theory with FEM.

According to CMT, the modal field distribution $F$ corresponding to the two supermodes in TCF with two identical cores is given by [22]

$$
F_{ \pm}(x, y)=\left(1+f_{ \pm}^{2}\right)^{-1 / 2}\left[F_{1}(x, y)+f_{ \pm} F_{2}(x, y)\right] .
$$

In the case of a symmetric TCF, $f_{ \pm}= \pm 1$ and the supermodes are the even and odd combinations of $F_{1}$ and $F_{2}$.

Additionally, the modal field distributions of the first-order supermode and the second-order supermode in the TCF can also be obtained accurately by the supermode theory with FEM calculations using the commercial COMSOL Multiphysics software.

Here same parameters are used for the TCF as those in the studies described in Section 2. The operational wavelength $\lambda$ is fixed at $1.55 \mu \mathrm{m}$, and the cladding refractive index $n_{0}$ is set to 1.444 . The values of the relative index difference $\Delta$, gap size $d_{g}$, and core radius $a$ are initially set to $0.25 \%, 2 \mu \mathrm{m}$, and $3.5 \mu \mathrm{m}$, respectively. For a strong coupling scenario with a particularly small gap size $d_{g}$, the modal field distributions of the first-order supermode and the second-order supermode in the TCF are plotted in Figure 5.

It is observed from Figure 5 that there is a discrepancy between the modal field distributions of the first-order supermode and the second-order supermode obtained by CMT and corresponding modal field distributions obtained by the supermode theory with FEM in the strong coupling scenario with a small gap size. To verify that the field distributions obtained by the two methods are different, the curves of the normalized electric field intensity for one-dimensional slices in three directions are depicted in Figure 6, and it is observed that the curves of the normalized electric field intensity obtained by CMT do not agree 


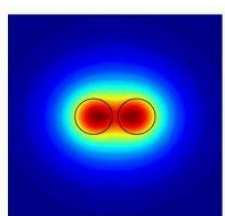

(a1)

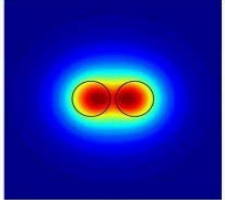

(a2)

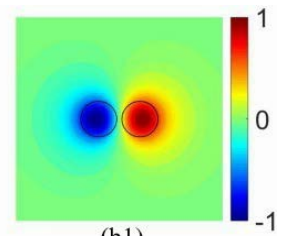

(b1)

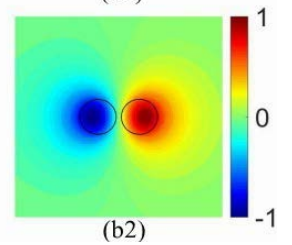

Figure 5. Modal distributions of the supermodes in the TCF with a gap size of $1 \mu \mathrm{m}$, core radius of $3.5 \mu \mathrm{m}$, with the wavelength of $1.55 \mu \mathrm{m}$, cladding index of 1.444 , and relative index difference of $0.25 \%$. (a1) 1st supermode obtained using CMT, (b1) 2nd supermode obtained using CMT, (a2) 1st supermode obtained by FEM calculations, (b2) 2nd supermode obtained by FEM calculations.
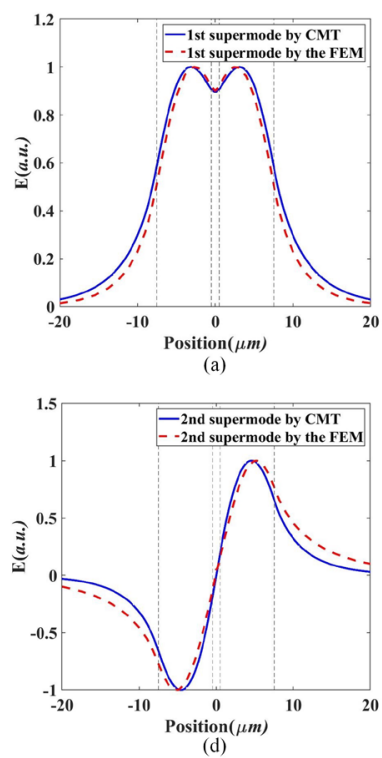

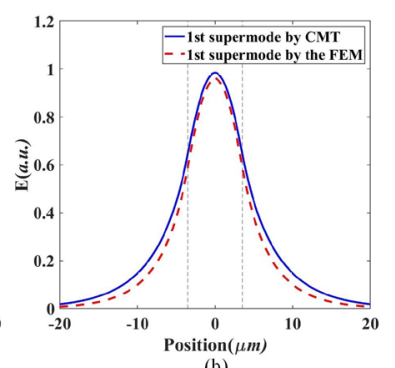

(b)

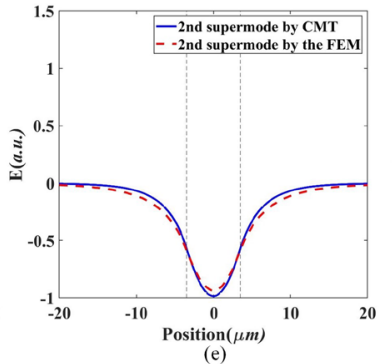

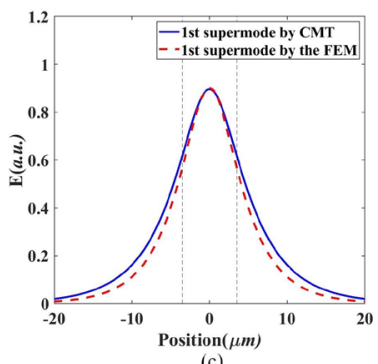

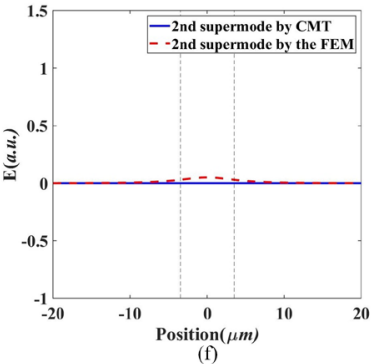

Figure 6. Two-dimensional modal field distribution of TCF with a gap size of $1 \mu \mathrm{m}$, core radius of $3.5 \mu \mathrm{m}$, working wavelength of $1.55 \mu \mathrm{m}$, cladding index of 1.444 , and relative index difference of $0.25 \%$. The dashed line in Fig represents the position of two cores. (a) 1 st supermode at $\mathrm{y}=0$, (b) 1 st supermode at $\mathrm{x}=-4 \mu \mathrm{m}$, (c) 1st supermode at $\mathrm{x}=0$, (d) 2nd supermode at $y=0$, (e) 2nd supermode at $x=-4 \mu \mathrm{m}$, (f) 2nd supermode at $\mathrm{x}=0$.

well with the results obtained by the FEM coupled supermode theory calculations.

Using the CMT and the supermode theory with FEM calculations, the modal field distributions of the first-order supermode and the second-order supermode in the TCF are simulated and the one-dimensional slices of normalized electric field intensities in three directions are also analyzed for a small core radius and a small relative refractive index difference. And it is shown that the simulation results are similar to those in the strong coupling scenario for a small gap size. The field distributions of the supermode in the TCF obtained by CMT are signifi- 
cantly different from the results obtained by the supermode theory with FEM in the strong coupling scenario for either a small core radius or a small relative refractive index difference.

In summary, the electric field intensities obtained by CMT that determine the coupling coefficients show clear errors in strong coupling scenarios such as when either the gap size, the core radius, or the relative refractive index difference is quite small. This is the origin of the obvious error obtained when Equation (4) based on CMT is used in the strong coupling scenario as mentioned in Section 2.

\section{Conclusion}

In this paper, the coupling coefficients are accurately calculated using the supermode theory with FEM approach. It is verified that the coupling coefficients obtained by the conventional analytical expression based on CMT have significant errors in strong coupling scenarios, affecting adversely the analysis of mode propagation for TCFs. In such scenarios, the more accurate results obtained by the supermode theory with FEM should be used. Furthermore, the modal field distributions that determine the coupling coefficients of the sueprmodes in a TCF with two identical single-mode cores are simulated using both CMT and the supermode theory with FEM. Simulation results show that the modal field distribution obtained by CMT is clearly inaccurate in the strong coupling scenario, revealing the origin of the differences between the two methods for calculating the coupling coefficients of TCFs.

\section{Conflicts of Interest}

The authors declare no conflicts of interest regarding the publication of this paper.

\section{References}

[1] Yuan, T., Zhang, X., Xia, Q., Wang, Y. and Yuan, L. (2020) A Twin-Core and Dual-Hole Fiber Design and Fabrication. J. Lightwave. Technol., 38, 1-5.

[2] Kowal, D., Urbanczyk, W. and Mergo, P.J.S. (2018) Twin-Core Fiber-Based Mach Zehnder Interferometer for Simultaneous Measurement of Strain and Temperature. Photonics, 18, 915. https://doi.org/10.3390/s18030915

[3] Lin, Z. and Changxi, Y. (2004) Polarization-Dependent Coupling in Twin-Core Photonic Crystal Fibers. J. Lightwave. Techno., 22, 1367-1373. https://doi.org/10.1109/JLT.2004.825356

[4] Wang, L., Shi, Z., Bai, Y., Lin, J. and Ge, Q.L. (2009) Influence of Cores' Shape on the Coupling Length of Dual-Core Fiber. 2009 Asia Communications and Photonics conference and Exhibition (ACP), Shanghai, 2-6 Nov. 2009, 1-2. https://doi.org/10.1364/ACP.2009.WL7

[5] Peterka, P., Kasik, I., Kanka, J., Honzatko, P., Matejec, V. and Hayer, M.J. (2000) Twin-Core Fiber Design and Preparation for Easy Splicing. IEEE Photonics. Technol. Lett., 12, 1656-1658. https://doi.org/10.1109/68.896339

[6] Zhang, S., Yuan, T. and Yuan, L. (2017) Asymmetrical Twin-Core Fiber Based Mi- 
chelson Interferometer for Environmental Refractive Index Sensing. 2017 25th Optical Fiber Sensors Conference (OFS), Jeju, 24-28 April 2017, 1-4. https://doi.org/10.1117/12.2264956

[7] Huang, L., Ren, G., Gao, Y., Zhu, B. and Peng, W. (2015) Design of Thermally Poled Twin-Core Fiber Coupler with Electrical Tunability. IEEE Photonics. Technol. Lett., 27, 919-922. https://doi.org/10.1109/LPT.2015.2401053

[8] Yang, K., He, J., Liao, C., Wang, Y., Guo, K., Liu, S. and Wang, Y. (2017) Twin-Core Few-Mode Fiber Bragg Gratings Inscribed by Femtosecond Laser. 2017 16th International Conference on Optical Communications and Networks (ICOCN), Wuzhen, 7-10 Aug. 2017, 1-3. https://doi.org/10.1109/ICOCN.2017.8121440

[9] Staszek, K., Kaminski, P., Rydosz, A., Wincza, K. and Gruszczynski, S. (2013) Miniaturized Broadband Three-Section Symmetrical Directional Coupler with Reduced Coupling Coefficient Requirements. IEEE MTT-S International Microwave and RF Conference, New Delhi, 14-16 Dec. 2013, 1-4.

https://doi.org/10.1109/IMaRC.2013.6777713

[10] Ankiewicz, A., Snyder, A. and Zheng, X.H. (1986) Coupling between Parallel OPTICAL Fiber Cores-Critical Examination. J. Lightwave. Technol., 4, 1317-1323. https://doi.org/10.1109/JLT.1986.1074906

[11] Liu, H., Fang, S. and Wang, Z. (2017) Trans-Directional Coupler with Adjustable Coupling Coefficients and Reconfigurable Responses. IET Microwaves, Antennas \& Propagation, 11, 1340-1346. https://doi.org/10.1049/iet-map.2016.0877

[12] Ivanov, N.V. and Korotkov, A.S. (2018) S-Band Microstrip Bandpass Filter Design Based on New Approach to Coupling Coefficients Calculation. 2018 IEEE International Conference on Electrical Engineering and Photonics (EExPolytech), St. Petersburg, 22-23 Oct. 2018, 60-63. https://doi.org/10.1109/EExPolytech.2018.8564442

[13] Mishin, S. and Oshmyansky, Y. (2017) Improving Coupling Coefficient Distribution on BAW Filters Manufactured on $200 \mathrm{~mm}$ Wafers. 2017 Joint Conference of the European Frequency and Time Forum and IEEE International Frequency Control Symposium (EFTF/IFCS), Besancon, 9-13 July 2017, 542-546. https://doi.org/10.1109/FCS.2017.8088952

[14] Ye, X.F., Zheng, S.Y. and Pan, Y.M. (2016) A Compact Millimeter-Wave Patch Quadrature Coupler with a Wide Range of Coupling Coefficients. IEEE. Microw. Wirel. Compon. Lett., 26, 165-167. https://doi.org/10.1109/LMWC.2016.2524581

[15] Hamidah, M., Rahardjo, S., Purnamaningsih, R.W., Poespawati, N.R. and Priambodo, P.S. (2017) Comparison of Coupling Coefficient Variation Effects on Double Couplers Structured Single Ring Resonator with Single and Double Beams Injection. 2017 15th International Conference on Quality in Research (QiR): International Symposium on Electrical and Computer Engineering, Nusa Dua, 24-27 July 2017, 357-361. https://doi.org/10.1109/QIR.2017.8168511

[16] Jang, S., Lee, Y. and Jae Su, Y. (2008) Coupling Coefficient Calculation of Laterally Coupled Distributed Feedback Laser Structure with Metal Surface Gratings. 2008 20 th International Conference on Indium Phosphide and Related Materials, Versailles, 25-29 May 2008, 1-3. https://doi.org/10.1109/ICIPRM.2008.4702918

[17] Okamoto, K. (2006) Charpter 4-Coupled Mode Theory. Fundamentals of Optical Waveguides, 2nd ed, Okamoto, Katsunari; Academic Press: Burlington, 159-207. https://doi.org/10.1016/B978-012525096-2/50005-2

[18] Jin, W. and Jian, S. (2017) Numerical and Simulation Analyses on Supermode Characteristics of Dual-Core Fiber and Four-Core Fiber. Optik, 32-38.

https://doi.org/10.1016/j.ijleo.2016.12.042 
[19] Chung Ho, K. and Kin Seng, C. (2002) Study of Polarization-Dependent Coupling in Optical Waveguide Directional Couplers by the Effective-Index Method with Built-In Perturbation Correction. J. Lightwave. Technol., 20, 1018-1026. https://doi.org/10.1109/JLT.2002.1018813

[20] Peng, G.D., Tjugiarto, T. and Chu, P.L.J.E.L. (2002) Polarisation Beam Splitting Using Twin-Elliptic-Core Optical Fibers. Electron. Lett., 26, 682-683. https://doi.org/10.1049/el:19900446

[21] Chan, F.Y., Lau, A.P.T. and Tam, H.Y. (2012) Mode Coupling Dynamics and Communication Strategies for Multi-Core Fiber Systems. Opt. Express., 20, 4548-4563. https://doi.org/10.1364/OE.20.004548

[22] Ren, W., Tan, Z. and Ren, G. (2015) Analytical Formulation of Supermodes in Multicore Fibers with Hexagonally Distributed Cores. IEEE Photonics J., 7, 1-11.

https://doi.org/10.1109/JPHOT.2015.2395993 